\title{
Modeling membranes under a transmembrane potential
}

\author{
Lucie Delemotte, François Dehez, Werner Treptow ${ }^{\S}$ and Mounir Tarek
}

UMR Structure et Réactivité des Systèmes Moléculaires Complexes, Nancy-University, CNRS, France

mtarek@edam.uhp-nancy.fr

\section{METHODS}

\section{Pure lipid membranes: (system A)}

The membrane model used for this study is an equilibrated fully hydrated palmitoyl-oleoylphosphocholine (POPC) bilayer. It consists of 288 lipid units and 14576 water molecules organized in two lamellae above and below the lipids. At the temperature set for the study, i.e. $30^{\circ} \mathrm{C}$, the bilayer is in the biologically relevant liquid crystal $\mathrm{L} \alpha$ phase. $140 \mathrm{Na}^{+}$and $140 \mathrm{Cl}^{-}$ ions are added to the solvent $(\sim 1 \mathrm{M})$. The final dimensions of the system before extending it in the $\mathrm{z}$ direction are $\sim 83 \times 92 \times 110 \AA^{3}$ and the total number of atoms is 65320 . Note that at this salt concentration the area per lipid had dropped from $62 \AA^{2}$ for the lipid bilayer without ions, to $\sim 54 \AA^{2}$, which is consistent with other simulations of lipid bilayers. ${ }^{1}$

Membranes containing the cyclic peptide nanotubes: (system B)

The systems are similar to those studied previously ${ }^{2,3}$. Nanotubes formed by eight cyclo[(LTrp-D-Leu) $)_{6}$ ] subunits were built using the "accelrys" visualization package InsightII. The choice of eight peptide subunits is rationalized by single-channel conductance experiments, 
which suggest for that particular sequence of amino acids that the number of rings be comprised between eight and ten. ${ }^{4}$ It also roughly coincides with the thickness of the hydrophobic core of the POPC bilayer. A cyclic peptide was constructed preliminarily, so that its carbonyl, $\mathrm{C}=\mathrm{O}$, and its amino, $\mathrm{N}-\mathrm{H}$, chemical bonds be normal to the plane of the ring. In such a cyclic structure, the conformation of the participating residues is that of a quasi $\beta$ strand. On account of the alternated L and D chirality, all side chains of the peptide point outwards. The $\chi$ torsional angles were chosen to warrant the flattest possible conformation of the ring. The cyclic peptides are stacked in such a fashion that (i) the L-Trp and the D-Leu side chains are distributed uniformly, and, (ii) eight inter-subunit backbone-backbone hydrogen bonds be formed between two consecutive rings. The backbone-backbone intermolecular hydrogen bonding interactions were optimized considering an antiparallel $\beta$-sheet-like stacked arrangement of the rings, in agreement with experimental observations. ${ }^{5,6}$ The geometry of the resulting cylindrical channel was finally optimized prior to insertion in the hydrated bilayer. The nanotubes were subsequently inserted at the center of the bilayer, with its longitudinal, principal axis parallel to the $z$-direction, normal to the interface. Overlapping lipid and water molecules were discarded to avoid poor van der Waals interactions. The final system contained 133 lipid molecules, 8280 water molecules and $39 \mathrm{Na}^{+}$and $39 \mathrm{Cl}^{-}$added to the water slabs, so that the concentration reaches $\sim 0.5 \mathrm{M}$. The dimensions of the system before the extension in the $\mathrm{z}$ direction are $80 \times 90 \times 110 \AA^{3}$.

Periodic boundary conditions were applied in all three directions of space.
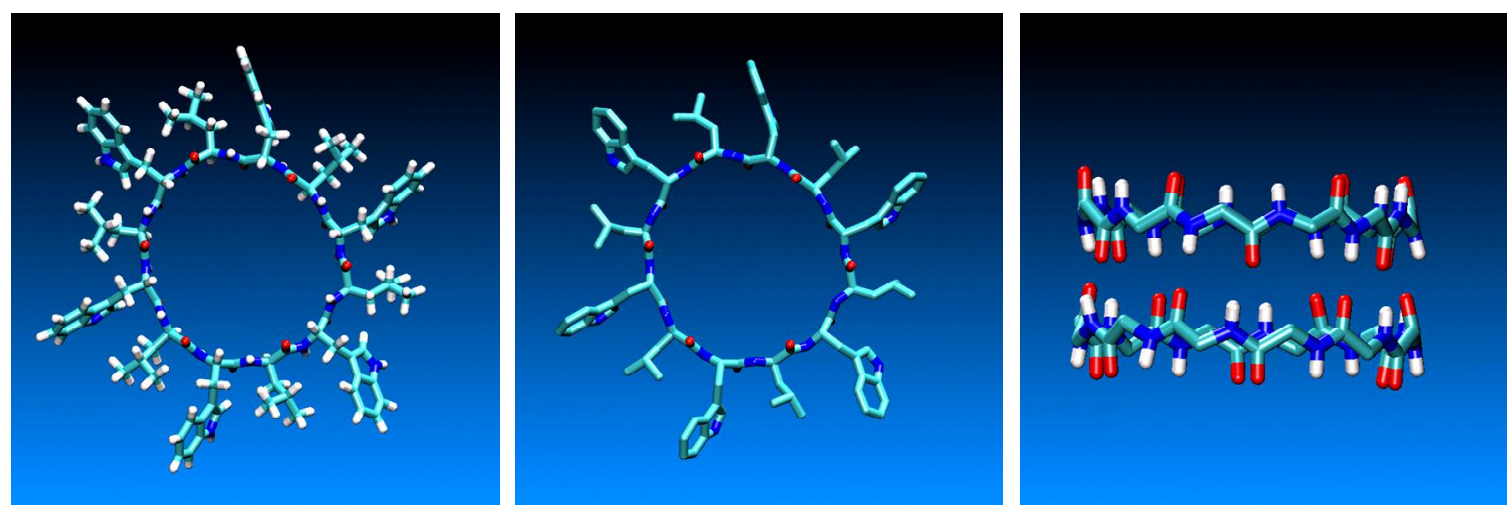

Figure 7: Conformation of the cyclo [(L-Trp-D-Leu $\left.)_{6}\right]$ peptides. On account of the alternated L and D chirality, all the side chains are pointing outward. Right: The cyclic peptide assemble 
through a network of intersubunit backbone-backbone hydrogen bonds between the oxygen atoms (red) of the carbonyl groups and the hydrogen atoms (white) of the amino groups.

\section{Molecular dynamics simulations.}

The MD simulation presented here was carried out using the program NAMD targeted for massively parallel architectures ${ }^{7,8}$. The systems were examined in the NPT (1atm and 300K) or NVT $(300 \mathrm{~K})$ ensembles employing the Langevin dynamics and Langevin piston method.

The equations of motion were integrated using the multiple time-step algorithm. A time-step of 2.0 fs was employed. Short- and long-range forces were calculated every 2 and 4 time-steps, respectively. Chemical bonds between hydrogen and heavy atoms were constrained to their equilibrium value. Long-range, electrostatics forces were taken into account using a fast implementation of the particle mesh Ewald (PME) approach ${ }^{9,10}$, with a direct space sum tolerance of $10^{-6}$ and a spherical truncation of $11 \AA$.

Bond stretching, valence angle deformation, torsional and non-bonded parameters of the cyclic peptides forming the nanotube, and non bonded interaction were all extracted from the all-atom CHARMM force field ${ }^{11}$. For the lipid (POPC) we used the united atom representation.

In order to maintain the structure of the nanotube, the distances between the nitrogen atoms of the amino moieties and the carbon atoms of the carbonyl groups pertaining to adjacent peptide rings were restrained to their equilibrium values by means of soft harmonic potential. ${ }^{3,12}$

\section{System set ups and simulations:}

All systems were first equilibrated at constant temperature $(300 \mathrm{~K})$ and constant pressure (1 Atm), as in the simulations of multi-lamella stacks of lipids. For the "charge imbalance" method, the system sizes were extended in the $\mathrm{z}$ direction $(\mathrm{Lz}=300 \AA)$ creating therefore an air water interface. Subsequent simulations were run at constant volume, using $3 \mathrm{~d}$ periodic boundary conditions, the thickness of the vacuum slab created above and bellow the solvent (70 $\AA$ each) being large enough to preclude significant interactions between the original cell 
and its replicas in the direction perpendicular to the bilayer. Five simulations of system A were performed, with charges imbalances $\mathrm{Q}$ going from 0 to $8 \mathrm{e}$, reaching a total of $7.5 \mathrm{~ns}$. For system $\mathrm{B}$, we carried out 10 simulations starting from higher charge imbalances $(\mathrm{Q}=8$ or $6 \mathrm{e})$, representing a total of $62 \mathrm{~ns}$.

Further simulations were performed using the standard "electric field method". In this case, a constant electric field $\overrightarrow{\mathrm{E}}$, normal to the bilayer, was applied on both "non-extended" systems, (i.e. original $\mathrm{MD}$ cell) and using $3 \mathrm{~d}$ periodic boundary conditions. For the membrane system $\mathrm{A}$, a total of 9 independent MD simulations performed at constant volume and constant temperature (total of $13 \mathrm{~ns}$ ) were generated under electric fields intensities ranging from 0.04 to $0.15 \mathrm{~V} \cdot \mathrm{nm}^{-1} .16$ independent simulations of system B containing the peptide nanotube (totaling $73 \mathrm{~ns}$ ) were performed at constant volume and constant temperature, under electric fields intensities ranging from 0.05 to $0.30 \mathrm{~V} . \mathrm{nm}^{-1}$. For the present systems, these electric fields generate (see below) transmembrane voltages ranging from 0.5 to $3 \mathrm{~V}$.

Electrostatic properties: The three-dimensional maps of the electrostatic potential characterizing the systems were generated by solving numerically the Poisson equation. This calculation was performed employing PMEPot module of the visualization program VMD. ${ }^{13}$ Instantaneous values of the electrostatic potential were averaged over 100-ps segments. The first derivative of the electrostatic potential was evaluated using OpenDX [http://www.opendx.org], an open-source visualization software package, to yield the threedimensional maps of the electric field. The electric properties of the molecular assemblies described in this contribution were visualized with OpenDX and the CMSP Chemistry module. $^{14}$ 


\section{RESULTS}

\section{A - Charge imbalance method}
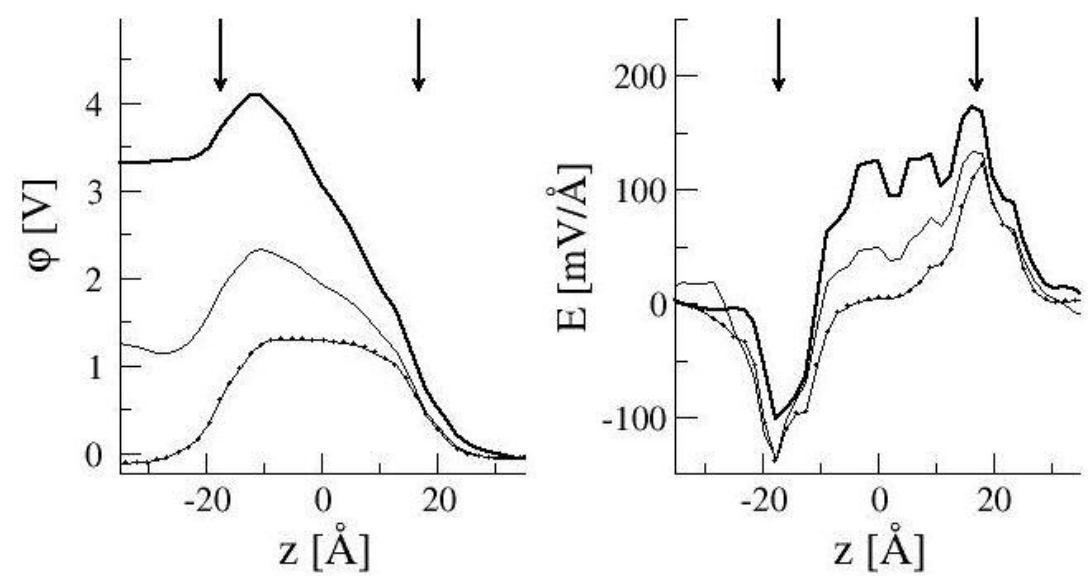

Figure 8: Left: Electrostatic potential profiles along the normal to the bilayer from simulations set at different transmembrane voltages. Right: corresponding electric field intensities. The arrows indicate the approximate position of the water membrane interface. Note that the electric field is oriented at each interface toward the electrolytes.

\section{B - Electric field method}
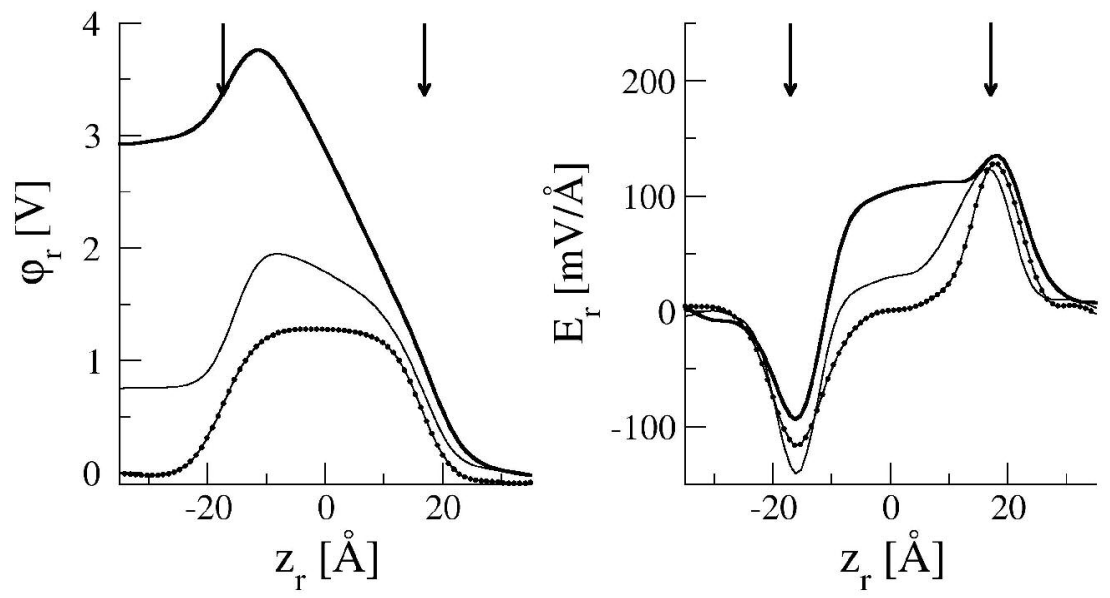

Figure 9: Electrostatic potential profiles along the normal to the bilayer estimated from the simulations of system A, generated with different applied electric fields (same as above). Left : corresponding electric field intensities. 


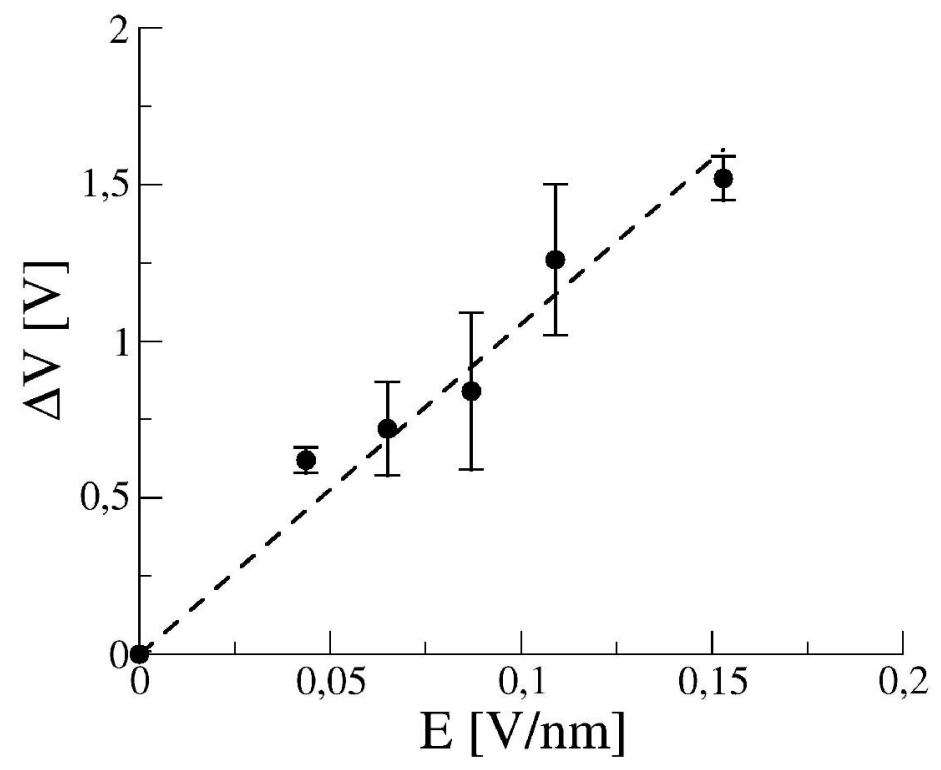

Figure 10: Transmembrane potential estimated from the MD simulations of the bilayer (system A), as a function of the intensity of the applied electric field. The error bars are standard deviations from the mean values estimates using blocks of $200 \mathrm{ps}$ trajectories. The dashed line represents the linear fit to these values, which slope is $10.5 \pm 0.5 \mathrm{~nm}$ ( about the size of the system in the $\mathrm{z}$ direction).

Note that as in simulations of bilayers at $0 \mathrm{M}$ salt concentration, the electric field $\overrightarrow{\mathrm{E}}$ induces a voltage difference over the whole system $\Delta \mathrm{V} \approx|\overrightarrow{\mathrm{E}}| \mathrm{L}_{z}$ where $\mathrm{L}_{z}$ is the size of the simulation box in the $\mathrm{z}$ direction.
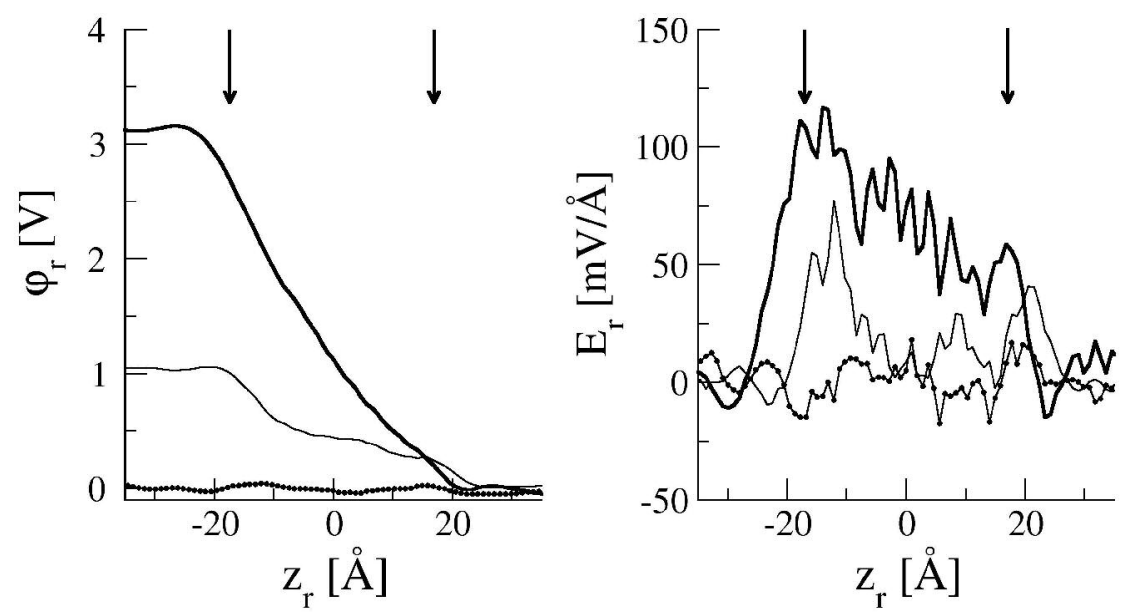

Figure 11: Left: Electrostatic potential profile estimated from the charge distributions along the nanotube axis for simulations of system B generated at different constant imposed electric 
fields of 0 (dots), 10 (thin line) and $30 \mathrm{mV} / \AA$ (thick line) Right: Corresponding electric field intensities.

Note that with this method, the net electric field felt by every charged particle during the MD simulation is the above electric field plus the applied field along $\mathrm{z}$.

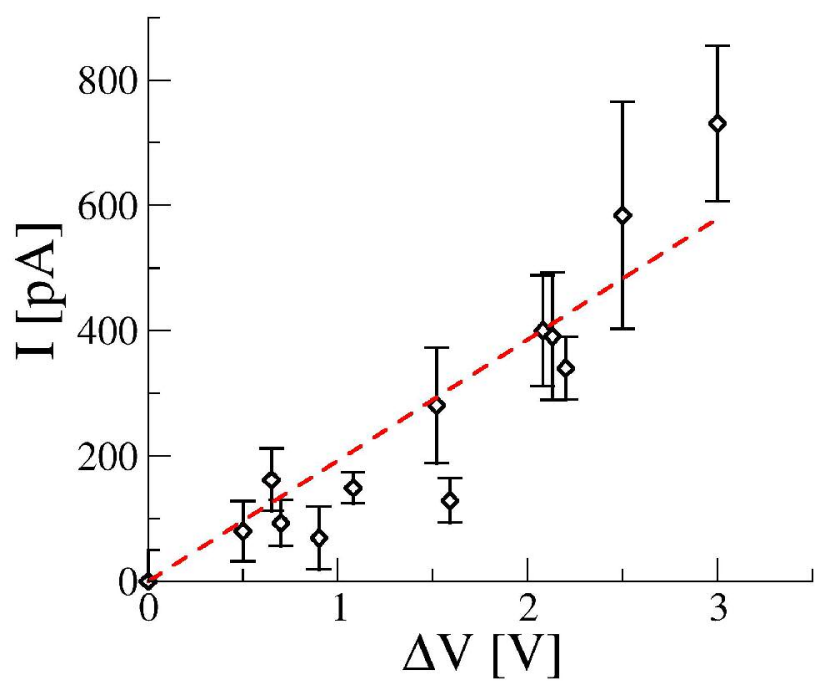

Figure 12: Intrinsic Na ionic currents estimated from the MD trajectories of system B performed under electric field, as a function of the resultant transmembrane voltage $\Delta \mathrm{V}$, estimated as $\Delta \mathrm{V} \approx|\overrightarrow{\mathrm{E}}| \mathrm{L}_{z}$. For each voltage (applied electric field) we report the mean value and the standard deviation estimated from several ions translocations (above 50). The dashed slope represents the values extracted from the "charge imbalance" method reported in Figure 6 of the main text.

Note that for the simulations performed at high electric field, $(\Delta \mathrm{V}=2.5 \mathrm{~V}$ and above), the intrinsic currents obtained from the Electric field method are higher than those obtained from the charge imbalance method.

\section{MOVIE CAPTION:}

Movie 1: MD simulation trajectory (initial $2 \mathrm{~ns}$ ) of the peptide channel (purple) imbedded in a hydrated POPC lipid bilayer, and subject to a TM voltage of $2 \mathrm{~V}$ generated by a charge imbalance between the lower and upper electrolytes. The system is at $500 \mathrm{mM} \mathrm{Na}^{+}$(yellow)- $\mathrm{Cl}^{-}$ (green) concentration, with $\mathrm{Q}=8 \mathrm{e}$ at $\mathrm{t}=0$. Color code: (head group atoms: $\mathrm{O}$ light green; $\mathrm{P}$ : gold; H: grey; N: C acyl chains: cyan). For clarity only the $\mathrm{O}$ of water molecules are represented (blue). Note the translocation of two $\mathrm{Na}+$ ions through the nanotube. 
Movie 2: Same as Movie1. Water is not represented.

Movie 3: Same as Movie1. Close up view.

Movie 4: MD simulation trajectory (initial $3 \mathrm{~ns}$ ) of the MthK bacterial potassium channel (purple) embedded in a membrane bilayer and subject to a TM potential (1V) generated by a charge imbalance between the upper and lower electrolytes. Note the translocation of the $2 \mathrm{~K}^{+}$ ions (gold) located in the selectivity filter (white) toward the extracellular side. The system is at $500 \mathrm{mM} \mathrm{K}^{+}$(yellow)- $\mathrm{Cl}^{-}$(green) concentration, with $\Delta \mathrm{V}=1 \mathrm{~V}$ at $\mathrm{t}=0$. For clarity water molecule are not represented. The lipid is in stick representation, and only two monomers of the MthK channel are represented (ribbon). (manuscript in preparation)

\section{References:}

(1) Cordomi, A.; Edholm, O.; Perez, J. J. J. Phys. Chem. B 2008, 112, 1397-1408.

(2) Tarek, M.; Maigret, B.; Chipot, C. Biophys. J. 2003, 85, 2287-2298.

(3) Dehez, F.; Tarek, M.; Chipot, C. J. Phys. Chem. B. 2007, 111, 10633-10635.

(4) Ghadiri, M. R.; Granja, J. R.; Buehler, L. Nature 1994, 369, 301-304.

(5) Ghadiri, M. R.; Granja, J. R.; Milligan, R. A.; McRee, D. E.; Khazanovich, N. Nature 1993, 366, 324-327.

(6) Hartgering, J. D.; Granja, J. R.; Milligan, R. A.; Ghadiri, M. R. J. Am. Chem. Soc. 1996, 118, 43-50.

(7) Kalé, L.; Skeel, R.; Bhandarkar, M.; Brunner, R.; Gursoy, A.; Krawetz, N.; Phillips, J.; Shinozaki, A.; Varadarajan, K.; Schulten, K. J. Comp. Phys. 1999, 151, 283-312.

(8) Bhandarkar, M.; Brunner, R.; Chipot, C.; Dalke, A.; Dixit, S.; Grayson, P.; Gullinsrud, J.; Gursoy, A.; Humphrey, W.; Hurwitz, D.; Krawetz, N.; Nelson, M.; Phillips, J.; Shinozaki, A.; Zheng, G.; Zhu, F. http://www.ks. uiuc.edu/Research/namd. 2002.

(9) Darden, T.; York, D.; Pedersen, L. J. Chem. Phys. 1993, 98, 10089-10092.

(10) Essmann, U.; Perera, L.; Berkowitz, M. L.; Darden, T.; Pedersen, L. G. J. Chem. Phys. 1995, 103, 8577-8593.

(11) MacKerell Jr., A. D.; Bashford, D.; Bellott, M.; Dunbrack Jr., R. L.; Evanseck, J.; Field, M. J.; Fischer, S.; Gao, J.; Guo, H.; Ha, S.; Joseph-McCarthy, D.; Kuchnir, L.; Kuczera, K.; Lau, F. T. K.; Mattos, C.; Michnick, S.; Ngo, T.; Nguyen, D. T.; Prodhom, B.; Reiher III, W. E.; Roux, B.; Schlenkrich, M.; Smith, J. C.; Stote, R.; Straub, J.; Watanabe, M.; WiorkiewiczKuczera, J.; Yin, D.; Karplus, M. J. Phys. Chem. B 1998, 102, 3586-3616.

(12) Asthagiri, D.; Bashford, D. Biophys. J. 2002, 82, 1176-1189.

(13) Humphrey, W.; Dalke, A.; Schulten, K. J. Molec. Graph. 1996, 14, 33--38.

(14) Gillilan, R. E.; Wood, F. Comput. Graphics 1995, 29, 55-58. 
